\title{
Photorealistic 3D Models and Interactive Learning Content for a Machine Elements E-Course
}

\author{
Petros Pistofidis, Department of Production Engineering and Management, Democritus University of Thrace, Greece \\ Pantelis N. Botsaris, Department of Production Engineering and Management, Democritus University of Thrace, Greece \\ Zacharias Giotsalitis, Department of Production Engineering and Management, Democritus University of Thrace, Greece
}

\begin{abstract}
Production and industrial engineering are domains that increasingly require a sophisticated and multidisciplinary set of skills. As modern educational mechanisms shift towards more advanced patterns of remote and asynchronous training, learning objects have emerged as an ideal application field for monitoring and evaluating the consumption of better composed and enriched content. This article discusses the ability of photorealistic 3D models and interactive 3D objects to support such competencies development and empower engineering perception. Introduced as a complementary dimension of video training, the research invests in photorealism and interactivity to allow the trainee experience with greater control and more detail the functional behavior of machine elements. Structured as exercise material for a lab of mechanical design, photorealistic 3D models were produced and embedded in an e-learning platform for engineering students to evaluate. Their feedback was captured through an extensive questionnaire, allowing for a deeper understanding of the approach's efficiency and results.
\end{abstract}

\section{KEYWORDS}

3D Modeling, E-Learning, Engineering Education, Machine Elements, Photo-Realism, Video Training

\section{INTRODUCTION}

Internet and modern technologies have upscaled the communication and connectivity potentials of everyone, making possible and quite affordable to access and consume high quality content from almost anywhere across the globe. Given how comfortable, direct and rich in terms of content video is, most people favor and have fully embraced it as their primary knowledge and information format. If a picture is worth a thousand words, a video has proved to be a much more powerful medium where images, pictures, photos and diagrams create dynamic knowledge contexts and convey information in a much more effective manner and at a significantly greater speed (Kay, 2012). Both commercial and academic use of high quality video assets provide constant validation that information perception and knowledge assimilation dramatically change when a story is being told visually (Orús et al., 2016). Storytelling engages learners and captures their attention inherently by the nature of the format. Each 
visual story is a stream of information the audience must buffer and process, while also translating and assessing the context of what has been learned in terms of purpose, goals and refined knowledge (Chtouki, Harroud, Khalidi, \& Bennani, 2012).

New multimedia technologies and techniques have provided a wide range of options and ways to upgrade the productivity of learning and teaching. Facilitating 3D visualizations and 3D models when creating educational material, is an established practice for a powerful learning experience with immersive environments and active roles inside them (Chau et al., 2013). 3D technologies are an integral part of modern digital assets and the platforms that host them. Their use is very common if not necessary for the development of soft skills and cognitive abilities that require better context understanding. Building virtual environments that accurately simulate critical events and scenarios is rapidly becoming the norm for hands-on experience in industry and other science domains where the cost and the complexity of providing real-case scenarios is forbidding (Bednarz, James, Widzyk-Capehart, Caris, \& Alem, 2015). The gamification of a learning process is augmented in every aspect, when the quality of the assets and the story is high. Providing a well-thought serious game requires mechanisms and content that motivates and fosters a feeling of accomplishment. 3D technologies now provide a platform and a medium for satisfying the learner, encouraging involvement and stimulating his or her critical thinking in complex tasks (Villagrasa, Fonseca, Redondo, \& Duran, 2014).

The potential of the provided 3D virtual environments and 3D objects lies primarily in the training of personnel that has the skills and background information to translate the visualized components into actionable knowledge. Modern engineering departments are currently familiarizing faculty and students with the concepts of Learning Factory (Baena, Guarin, Mora, Sauza, \& Retat, 2017) and its modern paradigms (Chryssolouris, Mavrikios, \& Rentzos, 2016). Adopting mechanisms that can instantiate these concepts, the presented research employs photorealism and interactivity to create a virtual training experience that offers the potentiality of better immersion without the cost and scheduling overhead of using expensive machinery units and physical mock-ups inside a classroom or a lab. Training through the use of 3D objects offers very tangible and competitive advantages over traditional methods of vocational education and training (VET):

- Enrolling in courses with consistent participation and interactive sessions. 3D training simulates real world experiences and this capturing process captivates trainees and increases retention.

- Trainees can navigate and explore events and spaces in a risk-free, but nevertheless realistic environment that encourages learning and ensures safe experimentation.

- Accelerated learning with leveled content consumption and longer unsupervised sessions adds up to a more cost-efficient allocation of resources, capitalizing in passive assets and allowing better allocation of human resources in key aspects of training.

- $3 \mathrm{D}$ virtual training is very effective in demonstrating interconnecting mechanisms and interfacing components inside sophisticated machinery that cannot be accessed or viewed by the human eye.

Industrial engineering training can gain a significant competitive edge from adopting the latest advances in learning technologies. This article presents the development process and the evaluation of a learning management system populated with 3D content that embodies modern photorealistic representations of digital assets. The delivery of the system was based on the open source Moodle Platform ${ }^{1}$ and the evaluation process provided evidence of positive acceptance by the academic and student communities. This article offers a brief analysis of the user evaluation feedback, drawing insight into the educational value of course structure and design choices, further leading to recommendations for improving the efficiency of composition and delivery of the training content. 


\section{BACKGROUND}

Modern industry is currently functioning inside a context of increasingly refined regulations and sophisticated guidelines. Adopting and incorporating advanced rules and specifications into a highly focused enterprise domain requires engineers that possess multidisciplinary skills that span from information and communication technology (ICT), to business and finance. Furthermore, solid knowledge of basic normative and legislative aspects is often required as a means to interface in crossfunctional teams and autonomous departments (Papathanassiou, Pistofidis, \& Emmanouilidis, 2013).

During the last decade e-Learning has reached significant maturity and gained even more popularity and ubiquity. Relevant research has pushed the boundaries towards a wide spectrum of directions, allowing asynchronous training and learning to earn its place as a rapidly adopted paradigm inside and outside academia and the workplace (Gros \& García-Peñalvo, 2016). Contributing to this, cloud technologies and enterprise social networks have provided the infrastructure and the context for the development of new learning tools and content, even more oriented and adapted to the learner and his or her needs (Zhang et al., 2015). Collaborative platforms and mobile learning services are now able to maintain and repurpose a constantly active link between the learner and an ever-growing pool of thematically organized and enriched knowledge. It is a link that invests on asynchronous interactivity, personalized experience and well-balanced modules of immersive content (MartínGutiérrez, Fabiani, Benesova, Meneses, \& Mora, 2015).

The cost of deploying and populating an e-Learning solution, inside an academic institution or an enterprise, can be much lower than that of managing and coordinating the assets and the process of conventional learning and training. The flexibility offered and the fact that e-Learning is by design an interactive and engaging experience, makes it appropriate for learners that require versatility, autonomy and the opportunity to access and consume more diverse and qualitative learning objects. Content and delivery are the main pillars of e-Learning, and their digital nature has allowed them to now reach a global audience in a convenient way and furthermore supplement most if not all providers of traditional learning (Arkorful \& Abaidoo, 2015).

One of the main advantages of on-line education is that a very large and diverse (from a point of access perspective) pool of learners can utilize the infrastructure and consume the digital assets of multiple repositories through a single virtual environment. The complexity of these assets has grown exponentially offering better linked knowledge and highly contextualized information (Okada et al., 2019). E-learning objects have evolved from page-to-page content with images and well-formatted text, to fully immersive experiences inside 3D virtual environments with augmented reality tools and interactions (Ramirez, Mendivil, Flores, \& Gonzalez, 2013). Video training, 3D visualizations and virtual labs constitute content dimensions that match and captivate the attention of modern learners. 3D modeling environments is a platform upon which simulation and case studies have built solid understanding and connection with learners' needs (Ewais \& De Troyer, 2016). Engineering students often have a much deeper understanding of complex structures and are commonly better qualified to consume such representations in a more efficient way. While their familiarity with such content is a facilitator to a higher level of scientific content comprehension, their expectations and needs grow accordingly (Martín-Gutiérrez, Mora, Añorbe-Díaz, \& González-Marrero, 2017).

The knowledge body currently offered in many e-Courses that serve the domain of Engineering Education is commonly structured in a thematically organized syllabus of videos and narrated presentations. Trading large bodies of texts and images with short and focused video content has been proven to better trigger the trainees' attention and maintain a more reliable learning context (Mayer, 2017). That is because such a context can be populated with much more vibrant, realistic and application-oriented content. Facilitating this, annotated and narrated videos manage to convey more information with sophisticated case studies and fully digitized experiences from industrial shop-floors, to highly congested production fields. 
Engineering students constitute a very challenging user to profile as a target learning group. They are early adopters of technology, constant followers of trending information and consumers of highly detailed media. Accustomed to such content, they have emerged as a more sophisticated audience to captivate and motivate with e-Learning content (Violante \& Vezzetti, 2015). While the ICT aspect of an e-Learning platform gains their attention and preference, the content often fails to meet their expectations in terms of media quality and interaction. In this paper, we explore how the leveraging of role, control and photorealism of 3D objects can benefit the experience of an engineering student inside an e-learning platform. In the next section we present the process of developing content that enhances the perception and clarity of how certain machine elements operate and fail. Delivered as e-Learning content that supports the exercises of a Mechanical Design Lab, the provided 3D models leverage quality, moving from detailed geometry and positioning to highly detailed textures and photorealism. Furthermore, we invest in tools that empower students to interactively handle 3D objects with annotations and select the best viewing angle that allows them to optimally understand the studied components and events.

\section{E-LEARNING OBJECT FOR MACHINE ELEMENTS}

The approach of the presented research is to build upon modern e-Learning tools and platforms and enrich them with content and interactivity that enhances the learning experience. Specifically, photorealism and materials have been used to create 3D models and animations that stimulate learners' attention and maintain their focus in understanding the operational behavior of machine elements at key stages of their life cycle. The visualizing environment offers viewing features that motivate interactivity and support learners to navigate, handle and study every aspect of the modeled elements and animated events.

\section{COURSE STRUCTURE AND MOODLE PLATFORM}

The content for two laboratory exercises was developed addressing failure mechanisms of specific machine elements. The exercises were implemented as e-courses in the form of a single page content syllabus. The goal was to engage engineering students with intuitive interface, modern media objects and exercise calculations. The course structure was organized with the following topics:

- Background Theory: A section that provides essential background for the course-related topics and discussion, including design parameters, component behavior and computation methodologies that populate the later sections of the course. It is supported by figures, graphs and tables.

- Exercise and Solution: This section provides initial data for the laboratory exercise and the system. It includes description and context information about the failure event and the studied mechanisms. The solution is available but initially hidden.

- Calculation Form: This section offers a form that calculates the exercise result and its purpose is to allow students test and monitor how different input values affect it. Each starting parameter has its own field and the output is colored accordingly, following the system's operational status. Links to additional resources that aid understanding, such as referencing tables, are available but initially hidden.

- Video Tutorials: The design schematics of the systems are provided at the beginning of this section. A series of video tutorials address the part of the exercise implementation that involves the use of SolidWorks ${ }^{2}$ software package. The assembly and simulation process are explained with detailed steps and instructions. The expected simulation report is available but initially hidden.

- 3D Viewer: This section includes the embedded 3D viewer for both the machine element models and the systems they operate in. This viewer offers the ability to move around the modelled object 
and view it from any desirable angle, close in and study the details of its textures or geometry, while also having access to annotations about key points and descriptive information.

- Photorealism (Animation and Images): A gallery of photorealistic images and animations is provided in this section. The images capture the system or components from angles that can help students identify important aspects of the failure event. The animations display the abnormal system operation that leads to element wear and eventually to its fault.

The delivered implementation is based on the widely adopted platform Moodle and facilitates appropriate extensions and plug-ins to embed code and integrate tools that render the following content:

- Rendering of complex mathematical equations and formulas.

- Video player for tutorials that reside inside the platform or at an external hosting service.

- Execution environment for code (JavaScript $\left.{ }^{3}\right)$ that calculates exercise results.

- Rendering mechanisms that allow the above code to dynamically change the formatting of course content and results.

- $3 \mathrm{D}$ viewer for an external cloud service $\left(\mathrm{Sketchfab}^{4}\right)$ that renders in real-time and supports the use of annotations.

- Gallery for high resolution renderings of 3D models that visualize failure events.

Usage of an advanced and well-established platform like Moodle has distinct benefits that couple with requirements and specifications of our research:

- Moodle offers an easy and versatile user and access control management, supporting the corresponding tasks and administrative actions with a full set of features incorporated into its core.

- Moodle constitutes a platform that simplifies and automates the process of frequent updates. Many of such updates are crucial for the fluid and fine-tuned operation of extensions and plugins that drive the enriched content of our e-courses.

- As a Web platform Moodle functions at a central point of installation, which in turn supports easy system backups, failure recovery and security configurations.

- Being an open source system is one of Moodle greatest strengths and advantages. Its structure and architecture foster customization and encourage new ways to create and display training modules.

- As a direct result of the former feature, Moodle has become a leading platform compatible with a rich and diverse pool of extensions and themes. This enables tailoring of both content and learning methodology, matching them to specific needs such as the ones of engineering students.

\section{Video Tutorials and Annotations}

Within the context of the proposed framework, annotated video tutorials represent core components of the learning process, as a widely adopted content type by most modern providers of e-training and e-learning courses. Media consumption of this type offers the baseline of user control and a tool where the user can interact with the content. The aim of their use in the proposed system is for the learner to follow steps and draw instructions in order to replicate a methodology and achieve the same result. The success of the video tutorials is based on two main elements, namely the virtual environment and the instructions.

- The virtual environment is the workspace where the targeted process is visualized by changing views and switching between content types that better convey each step. It is the main visual canvas and allows the presentation of complex scenes and graphs, including kinematics, plant layouts, and multimedia components. For the content of the machine elements courses, the virtual environment was the workspace of the SolidWorks software package. The tutorials describe a 
specific workflow of actions the students must complete in order to successfully produce the models for components such as machinery bolts and belts.

- The annotated instructions operate as pointers to the objects populating the virtual workspace. The visualized process is enriched with a second layer of content that represents milestones, progress structure, critical points and many other semantic means that learners understand, consume and orientate themselves more efficiently in the virtual workspace. For the presented research, annotations were used to indicate specific interface components of the virtual environment and narrate the process that was visualized. Annotations also allowed the display of bullet-points, the students had to keep track of and remember in order to successfully configure or design a component with reference to a larger system.

Both the quality of the virtual environment and the annotated instructions are tightly connected with the underlying learning management system and its ability to embed and fully support updated and fully featured media players. Facilitating the appropriate plugins and platform extensions, the proposed system was able to effectively stream and play large and high-quality media files that reside both in cloud storage (Google Drive ${ }^{5}$ ) and popular hosting platforms (YouTube ${ }^{6}$ ). The players provided a smooth viewing experience with support for annotations and timestamps. The use of familiar Web services and tools resulted in better user retention and longer sessions. Composing more videos of smaller duration was a course development decision that gave better control to the student and the author. The small video format requires balanced attention span and intensity, while also allowing for better segmentation and handling of knowledge topics.

\section{Selective Viewing and Calculation Forms}

It is a common format of modern LMS to provide single page courses with all the material included, linked or hidden inside one single page. The referencing nature of web content and the dynamic viewing capabilities of the technologies that power its rendering, allow the easy and effective navigation of even the most complex content structures. A single page course may provide a sequence of video tutorials that are fully supported by descriptive summaries, taxonomy tags and other useful information. Supporting this experience with balanced interactivity and feedback is a key point for assessing how the content is measuring up to the users' expectations and whether it is effectively consumed.

Latest course implementations and the presented research follow a diversifying paradigm that blends the viewing sessions with user interactivity and does so with interface mechanisms that provide instant access to forms and hidden content that the learner has the option to use. Providing consistently the same format of educational material inside a single page course will eventually demystify any advantages it has to offer. Cycling through content and input that require a different level of attention and processing, will not exhaust the user and instead will allow him or her to better value the quality of the course material.

With selective viewing the learner can trigger the display of hidden content in our system. Using interface components as triggers, the user can access links to download files, embedded players for videos and embedded viewers for specific document types. Each such trigger also acts as a confirmation or a flag that validates the user's interest in learning more about the subject, seeking the solution to a problem, or prompting for access to external resources and further reading.

Calculation forms act as a self-assessment tool that learners can use to cross examine results and confirm the correct completion of the exercise's computations. They were developed using web technologies and the produced code was able to run inside a Moodle extension that enables its controlled and monitored execution. Each course's respective form provides a way to easily check the result for multiple sets of input parameters. This helps identify and map the input sets that produce results beyond the threshold of certain failure events. Furthermore, given that the code operates directly in the context of the course, it is possible to reuse it in future mechanical design exercises that study multi-staged scenarios of failure events. 
Figure 1. 3D model viewer displaying belt and electric motor with annotations

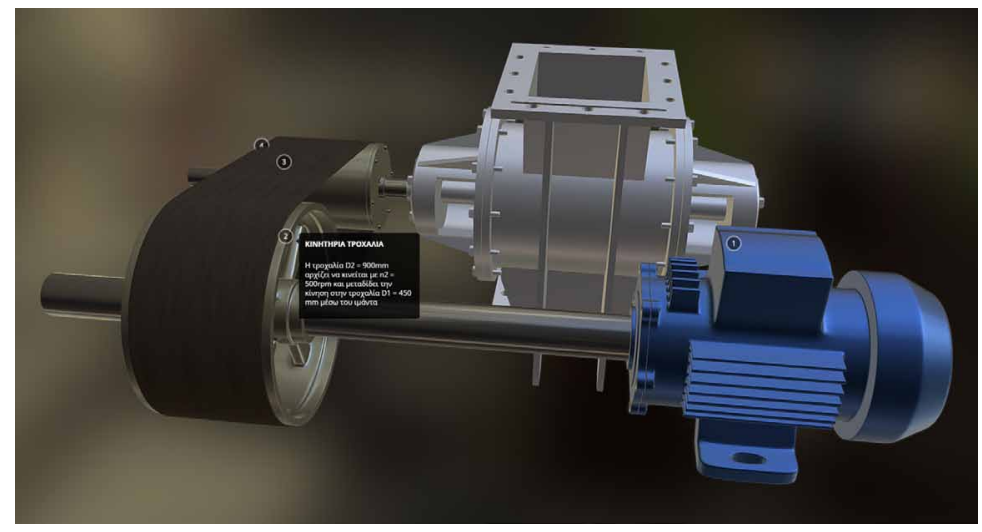

\section{Photorealism and Interactive 3D Viewer}

Creating a virtual environment, where engineering students can experience how machinery operate and fail with high fidelity visual representations, is a key target of the presented research. The produced content includes accurate 3D models of machinery elements placing them as parts of larger systems and machinery units. Animations were also designed and produced displaying the failure events described in each laboratory exercise. In greater detail the 3D digital assets visualize:

Case A: The failure of a belt as part of an electric motor engine.

Case B: The failure of a bolt as part of a steam generator.

Both models were made available to view, handle and navigate in each course through a Web application that can render them in a real time manner (Figure 1). The delivered e-courses include a viewer of the 3D models through the use of a customized Moodle module for the Sketchfab web services (Figure 2). The e-courses also provide photorealistic animations and stills (Figure 3, Figure 4) for the following knowledge:

- The assembly of the studied machinery unit with all machine elements coming together to connect and interface.

- The normal system behavior with machine elements operating under stress.

- The mechanisms that take place and the failure event, when machine elements reach their stress limits.

Annotations essentially formulate a layer of metadata that describe the 3D visualized knowledge. Starting with simple tags for glossary terms and video highlights, a new level of complexity is introduced. Annotations that refer to objects that are temporal in nature, such as a training video, can be considered as links that are fluidly constructed, visualized and navigated. In the produced 3D models and animations, the incorporated annotations present information about parameters and components that play a critical role in the normal operation and the studied failure events. For the case of the 3D rendering viewer these annotations constitute active interface triggers that reposition the camera and provide a better viewing angle of the annotated machine element.

Browsing photorealistic models and consuming the annotated information is the first step towards a larger use of 3D technologies linking academia and industry with training repositories of high-quality digital assets. Since the point-of-access of such virtual environments can easily become 
Figure 2. 3D model viewer displaying bolt and steam generator with player interface
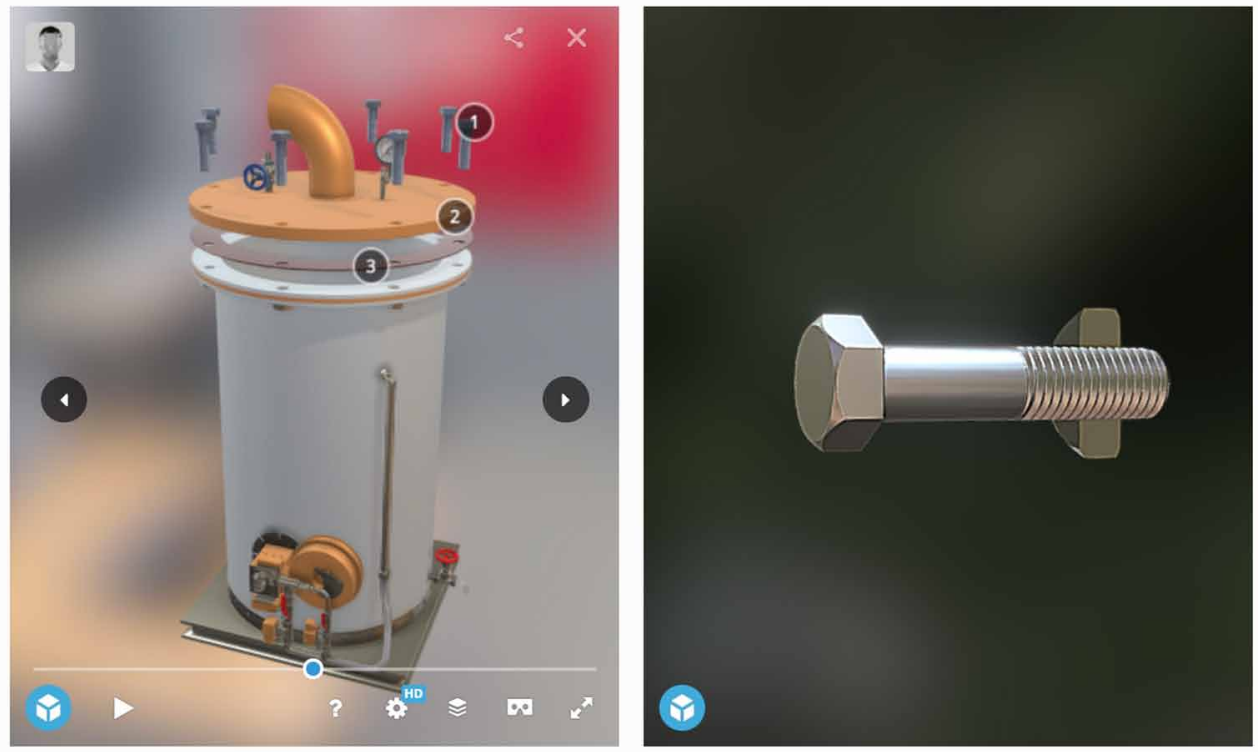

Figure 3. Photorealistic still image of the belt and motor

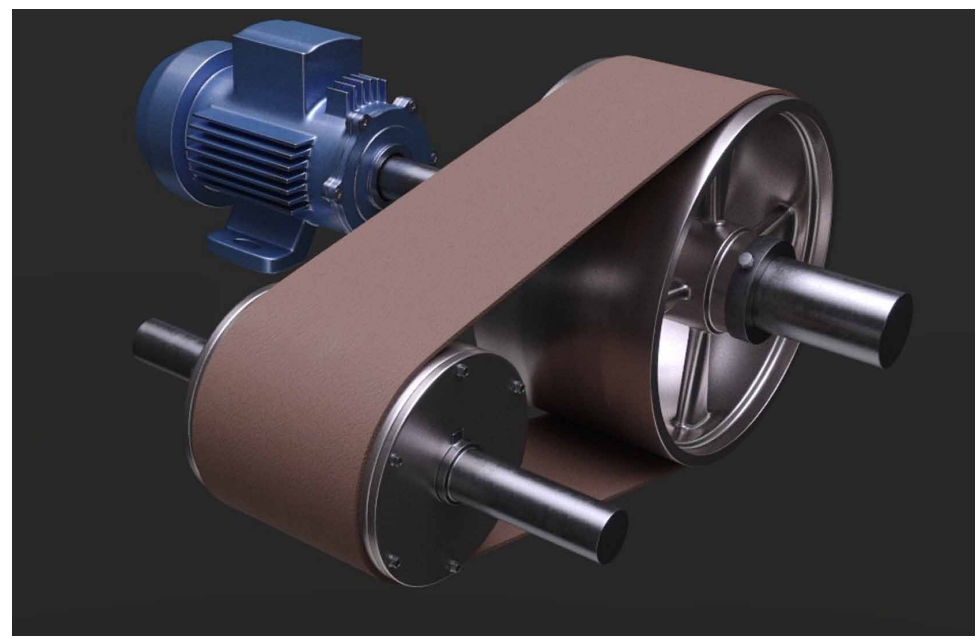

anyplace outside the classroom or the production field, their services can be invoked by specialized roles and profiled events that originate from the engineering domain. A platform such as Moodle provides an environment where engineering professionals and students communicate and collectively annotate and study immersive visualizations of failure events and maintenance practices. What is currently organized as e-courses for a Laboratory of Mechanical Design, can easily scale to integrate 
Figure 4. Photorealistic rendering of the bolts and steam generator

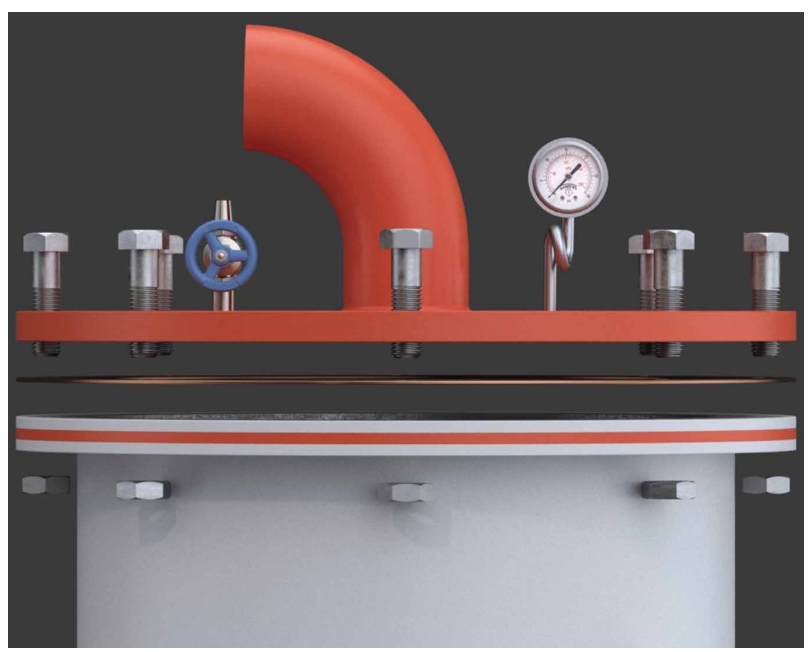

3D models, technical manuals, photographs, audios and videos that enhance an extremely accurate $3 \mathrm{D}$ representation of products and plants for training, every-day and extra-ordinary maintenance.

Making such visualizations, for a variety of failures and normal operations, available to everyday access can develop important perception and analytical skills that students could hardly acquire even in real industrial shop-floors. Allowing this type of knowledge to be asynchronously accessed by engineers and customized in a personalized manner, can lead to even more tangible benefits, simply through the filtering of digital assets and the real-time compilation of immersive scenarios. The delivered platform is available on-line ${ }^{7}$ and visitors can access and view the produced content with the provided user credentials.

\section{CONCLUSION}

Engineering students have a positive perception of e-learning tools and modern media types. System features such as usability, navigation efficiency and visually appealing content, all contribute to what they expect as an engaging learning experience. Populating content pages with videos, 3D models and animations creates an environment tuned for learning. Single page course appearance provides speedy content delivery and supports flexible navigation. The delivered content and learning platform were evaluated by engineering students and their feedback captured using a questionnaire comprising questions about:

Set I: the use of media and 3D content in e-learning platforms they access and use.

Set II: the content quality, the navigation efficiency and the overall usability of the pilot system.

Set III: the added value of media content for the pilot system.

Set IV: the added value from using 3D technologies for the content of the pilot system.

Set V: proposed features and improvements.

The questionnaire starts with exploring how participants evaluate the availability of 3D objects in other training material and their feedback described this availability as lacking, with static and nondynamic exceptions. They reported that most of the media content in e-learning platforms constitute narrated slide-based presentations without the support of advanced visualizations. Then participants were called to rate the quality and contribution of the produced $3 \mathrm{D}$ models in the learning experience. 
More than $60 \%$ rated the quality of 3D models and animations as "Very Good", pinpointing the impact of photorealism as a quality feature that was expected and much appreciated. When prompted to rank the importance and added value of photorealism, almost $70 \%$ of them responded with "Very Important". Many students reported that photorealism is a default feature of the media content they consume on a daily base, during entertainment or other activities. Not being able to experience a somehow similar level of immersion and realism, when consuming educational media, creates a growing sense of lackluster quality and a gap of enthusiasm. Proposed new features included placing the $3 \mathrm{D}$ assets inside a virtual reality lab and interacting with them using controllers. It is worth mentioning that the 3D rendering service that was used in the pilot system, currently supports virtual reality headsets.

Frameworks and tools that build 3D virtual spaces in Web environments have evolved through the use of cloud and Web technologies and are now able to efficiently integrate with advanced e-Learning systems. 3D photorealistic content can accurately communicate design details and simulate the visual stimulus of real processes. Such features trigger students' imagination and allow them to fuse knowledge from the design thinking and the life-cycle condition state of multiple engineering objects that could never otherwise be viewed or compared. This article has looked into the use of photorealistic 3D models and their supporting interactive viewing tools, as content modules for an e-Learning platform that support a growing curricula of lab exercises for Machine Elements. The research concludes that immersive 3D models offer a learning content type that increases flexibility, knowledge depth and cost-efficiency. The authors strongly believe that both the development and assessment of engineering competence and design skills constitute fields where virtual training with immersive environments and objects can outperform traditional e-training solutions and provide a solid platform for Learning Factories. 


\section{REFERENCES}

Arkorful, V., \& Abaidoo, N. (2015). The role of e-learning, advantages and disadvantages of its adoption in higher education. International Journal of Instructional Technology and Distance Learning, 12(1), 29-42.

Baena, F., Guarin, A., Mora, J., Sauza, J., \& Retat, S. (2017). Learning factory: The path to industry 4.0. Procedia Manufacturing, 9, 73-80. doi:10.1016/j.promfg.2017.04.022

Bednarz, T., James, C., Widzyk-Capehart, E., Caris, C., \& Alem, L. (2015). Distributed collaborative immersive virtual reality framework for the mining industry. In Machine Vision and Mechatronics in Practice. Springer.

Chau, M., Wong, A., Wang, M., Lai, S., Chan, K. W., Li, T. M., Chu, D., Chan, I. K. W., \& Sung, W. (2013). Using 3D virtual environments to facilitate students in constructivist learning. Decision Support Systems, 56, 115-121. doi:10.1016/j.dss.2013.05.009

Chryssolouris, G., Mavrikios, D., \& Rentzos, L. (2016). The teaching factory: A manufacturing education paradigm. Procedia CIRP, 57, 44-48. doi:10.1016/j.procir.2016.11.009

Chtouki, Y., Harroud, H., Khalidi, M., \& Bennani, S. (2012). The impact of YouTube videos on the student's learning. Paper presented at the 2012 International Conference on Information Technology Based Higher Education and Training (ITHET). doi:10.1109/ITHET.2012.6246045

Ewais, A., \& De Troyer, O. (2016). Authoring adaptive 3D virtual learning environments. In Web Design and Development: Concepts, Methodologies, Tools, and Applications. IGI Global.

Gros, B., \& García-Peñalvo, F. J. (2016). Future trends in the design strategies and technological affordances of e-learning. Learning, Design, and Technology: An International Compendium of Theory, Research, Practice, and Policy, 1-23.

Kay, R. H. (2012). Exploring the use of video podcasts in education: A comprehensive review of the literature. Computers in Human Behavior, 28(3), 820-831. doi:10.1016/j.chb.2012.01.011

Martín-Gutiérrez, J., Fabiani, P., Benesova, W., Meneses, M. D., \& Mora, C. E. (2015). Augmented reality to promote collaborative and autonomous learning in higher education. Computers in Human Behavior, 51, 752-761. doi:10.1016/j.chb.2014.11.093

Martín-Gutiérrez, J., Mora, C. E., Añorbe-Díaz, B., \& González-Marrero, A. (2017). Virtual technologies trends in education. Eurasia Journal of Mathematics, Science and Technology Education, 13(2), 469-486.

Mayer, R. E. (2017). Using multimedia for e-learning. Journal of Computer Assisted Learning, 33(5), 403-423. doi:10.1111/jcal.12197

Okada, Y., Haga, A., Wei, S., Ma, C., Kulshrestha, S., \& Bose, R. (2019). E-Learning Material Development Framework Supporting 360VR Images/Videos Based on Linked Data for IoT Security Education. Paper presented at the International Conference on Emerging Internetworking, Data \& Web Technologies. doi:10.1007/978-3030-12839-5_14

Orús, C., Barlés, M. J., Belanche, D., Casaló, L., Fraj, E., \& Gurrea, R. (2016). The effects of learner-generated videos for YouTube on learning outcomes and satisfaction. Computers \& Education, 95, 254-269. doi:10.1016/j. compedu.2016.01.007

Papathanassiou, N., Pistofidis, P., \& Emmanouilidis, C. (2013). Competencies development and self-assessment in maintenance management e-training. European Journal of Engineering Education, 38(5), 497-511. doi:10. 1080/03043797.2013.811475

Ramirez, H., Mendivil, E. G., Flores, P. R., \& Gonzalez, M. C. (2013). Authoring software for augmented reality applications for the use of maintenance and training process. Procedia Computer Science, 25, 189-193. doi:10.1016/j.procs.2013.11.023

Villagrasa, S., Fonseca, D., Redondo, E., \& Duran, J. (2014). Teaching case of gamification and visual technologies for education. Journal of Cases on Information Technology, 16(4), 38-57. doi:10.4018/ jcit.2014100104 
Violante, M. G., \& Vezzetti, E. (2015). Virtual interactive e-learning application: An evaluation of the student satisfaction. Computer Applications in Engineering Education, 23(1), 72-91. doi:10.1002/cae.21580

Zhang, X., Gao, Y., Yan, X., de Pablos, P. O., Sun, Y., \& Cao, X. (2015). From e-learning to social-learning: Mapping development of studies on social media-supported knowledge management. Computers in Human Behavior, 51, 803-811. doi:10.1016/j.chb.2014.11.084

\section{ENDNOTES}

Moodle Platform - https://moodle.org

SolidWorks - https://www.solidworks.com/

JavaScript - https://www.w3schools.com/js/

Sketchfab - https://sketchfab.com/

Google Drive - https://www.google.com/drive/

YouTube - https://www.youtube.com/

MeDiLab PME-Moodle - https://medilab.pme.duth.gr/13-en.shtml

Petros Pistofidis (PhD, MSc) received his Diploma in Computer Engineering and Informatics from the University of Patras (Greece), in 2005. In 2006 he completed his MSc in Computer Science from the University of Edinburgh (UK), majoring in Computer Systems and Software Engineering. In 2016 he successfully supported his PhD thesis in Technologies and Systems for Remote Monitoring and Diagnosis Support, at the School of Engineering of Democritus University of Thrace (DUTH - Greece). He is currently a Post-Doc Researcher at the Production Engineering and Management Department of DUTH. His research interests include e-Maintenance, reliability centered maintenance, internet of things, as well as enterprise, mobile and context-aware computing.

P. N. Botsaris is Former Deputy Rector of Research and Innovation of Democritus University of Thrace (DUTH) as well as Former Head of the University's Research Committee (SARF) responsible for the research and development of the university from 2013 to 2018. He is Professor at the Department of Production Engineering and Management, DUTH in the dield of the Mechanical Design. He studied at the School of Engineering, Department of Electrical and Computer Engineering, Faculty of Energy Systems, Lab. of Mechanical Engineering, Democritus University of Thrace. He holds two National Patents from the Industrial Property Organisation of Hellas (O.B.I.). He has participated as a scientific coordinator and main researcher in many EU and national projects and is actively involved in innovative product development and promotion of entrepreneurship and business growth. He was the Scientific Coordinator of the Innovation and Entrepreneurship Unit of the Democritus University of Thrace that aims at the development of the entrepreneurial culture and thinking of students and graduates, from 2012 to 2018 . He is author of more than 100 scientific papers in international journals and conferences.

Zacharias Giotsalitis received his Diploma in Product and Management Engineering from Democretus University of Thrace (Greece), in 2017. His research interests include Computer-aided Design and Photorealistic 3D Modeling. He is currently working on the field of $3 D$ visualization for architectural projects. 